ELECTRONIC RESEARCH ANNOUNCEMENTS OF THE AMERICAN MATHEMATICAL SOCIETY

Volume 13, Pages 53-59 (June 11, 2007)

S $1079-6762(07) 00174-6$

\title{
A NATURAL SMOOTH COMPACTIFICATION OF THE SPACE OF ELLIPTIC CURVES IN PROJECTIVE SPACE
}

\author{
RAVI VAKIL AND ALEKSEY ZINGER
}

(Communicated by János Kollár)

\begin{abstract}
The space of smooth genus-0 curves in projective space has a natural smooth compactification: the moduli space of stable maps, which may be seen as the generalization of the classical space of complete conics. In arbitrary genus, no such natural smooth model is expected, as the space satisfies "Murphy's Law". In genus 1, however, the situation remains beautiful. We give a natural smooth compactification of the space of elliptic curves in projective space, and describe some of its properties. This space is a blowup of the space of stable maps. It can be interpreted as a result of blowing up the most singular locus first, then the next most singular, and so on, but with a twist - these loci are often entire components of the moduli space. We give a number of applications in enumerative geometry and Gromov-Witten theory. For example, this space is used by the second author to prove physicists' predictions for genus-1 Gromov-Witten invariants of a quintic threefold. The proof that this construction indeed gives a desingularization will appear in a subsequent paper.
\end{abstract}

\section{Desingularization of the moduli space of Genus-one maps}

The moduli space of stable maps $\overline{\mathcal{M}}_{g, k}(X, \beta)$ to a complex projective manifold $X$ (where $g$ is the genus, $k$ is the number of marked points, and $\beta \in H_{2}(X, \mathbb{Z})$ is the image homology class) is the central tool and object of study in GromovWitten theory. We regard this space as a Deligne-Mumford stack. The open subset corresponding to maps from smooth curves is denoted $\mathcal{M}_{g, k}(X, \beta)$.

The paradigmatic example is $\overline{\mathcal{M}}_{0, k}\left(\mathbb{P}^{n}, d\right)$. This space is wonderful in essentially all ways: it is irreducible, smooth, and contains $\mathcal{M}_{0, k}\left(\mathbb{P}^{n}, d\right)$ as a dense open subset. The boundary

$$
\Delta:=\overline{\mathcal{M}}_{0, k}\left(\mathbb{P}^{n}, d\right) \backslash \mathcal{M}_{0, k}\left(\mathbb{P}^{n}, d\right)
$$

is normal crossings. The divisor theory is fully understood, and combinatorially tractable, $[\mathrm{P}$. In some sense, this should be seen as the natural generalization of the space of complete conics compactifying the space of smooth conics.

It is natural to wonder if such a beautiful structure exists in higher genus. In an arbitrary genus, however, there is no reasonable hope: even the interior $\mathcal{M}_{g}\left(\mathbb{P}^{n}, d\right)$ is badly behaved in general. More precisely, $\mathcal{M}_{g}\left(\mathbb{P}^{n}, d\right)$ (as $g, n$, and $d$ vary) is

Received by the editors July 13, 2006.

2000 Mathematics Subject Classification. Primary 14D20; Secondary 53D99.

The first author was partially supported by PECASE/CAREER grant DMS-0238532. The second author was partially supported by a Sloan Fellowship and NSF Grant DMS-0604874.

(C)2007 American Mathematical Society 
arbitrary singular in a well-defined sense -it may have essentially any singularity, and can have components of various dimension meeting in various ways with various nonreduced structures [V2]. There is no reasonable hope for describing a desingularization, as this would involve describing a resolution of singularities.

In genus one, however, the situation remains remarkably beautiful. Although $\overline{\mathcal{M}}_{1, k}\left(\mathbb{P}^{n}, d\right)$ in general has many components, it is straightforward to show that $\mathcal{M}_{1, k}\left(\mathbb{P}^{n}, d\right)$ is irreducible and smooth. Let $\overline{\mathcal{M}}_{1, k}^{0}\left(\mathbb{P}^{n}, d\right)$ be the closure of this open subset (the "main component" of the moduli space).

In the paper [VZ], we will show that there is a natural desingularization of this main component

$$
\widetilde{\mathcal{M}}_{1, k}\left(\mathbb{P}^{n}, d\right) \rightarrow \overline{\mathcal{M}}_{1, k}^{0}\left(\mathbb{P}^{n}, d\right)
$$

This desingularization has several desirable properties.

- It leaves the interior $\mathcal{M}_{1, k}\left(\mathbb{P}^{n}, d\right)$ unchanged.

- The boundary $\widetilde{\mathcal{M}}_{1, k}\left(\mathbb{P}^{n}, d\right) \backslash \mathcal{M}_{1, k}\left(\mathbb{P}^{n}, d\right)$ is simple normal crossings, with an explicitly described normal bundle.

- The points of the boundary have an explicit geometric interpretation.

- The desingularization can be interpreted as blowing up "the most singular locus", then "the next most singular locus", and so on, but with an unusual twist.

- The divisor theory is explicitly describable, and the intersection theory is tractable. (For example, one can compute the top intersection of any combination of divisors using $\mathrm{Z} 2$.)

- The compactification is natural in the following senses.

(i) The desingularization is equivariant - it behaves well with respect to the symmetries of $\mathbb{P}^{n}$. Hence we can apply Atiyah-Bott localization to this space - not just in theory, but in practice.

(ii) It behaves well with respect to the inclusion $\mathbb{P}^{m} \hookrightarrow \mathbb{P}^{n}$.

(iii) It behaves well with respect to the marked points (forgetful maps; $\psi$-classes; etc.).

(iv) Consider the universal map

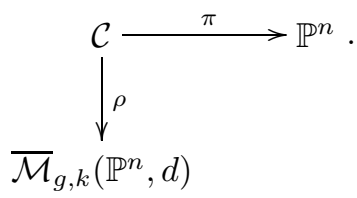

An important sheaf in Gromov-Witten theory is $\rho_{*} \pi^{*} \mathcal{O}_{\mathbb{P}^{n}}(a)$. When $g>0$, this is not a vector bundle, which causes difficulty in computation and theory. However, in genus 1, "resolving $\overline{\mathcal{M}}_{1, k}^{0}\left(\mathbb{P}^{n}, d\right)$ also resolves this sheaf": when the sheaf is pulled back to the desingularization, it "becomes" a vector bundle. More precisely: it contains a natural vector bundle, and is isomorphic to it on the interior. This vector bundle is explicitly describable.

We think it is interesting that such a natural naive approach as we describe below actually works, and yields a desingularization with these nice properties. For example, if $n>2$, this desingularization can be interpreted as a natural compactification of the Hilbert scheme of smooth degree $d$ genus- 1 curves in projective space, and thus could be seen as the genus- 1 version of the complete conics. 


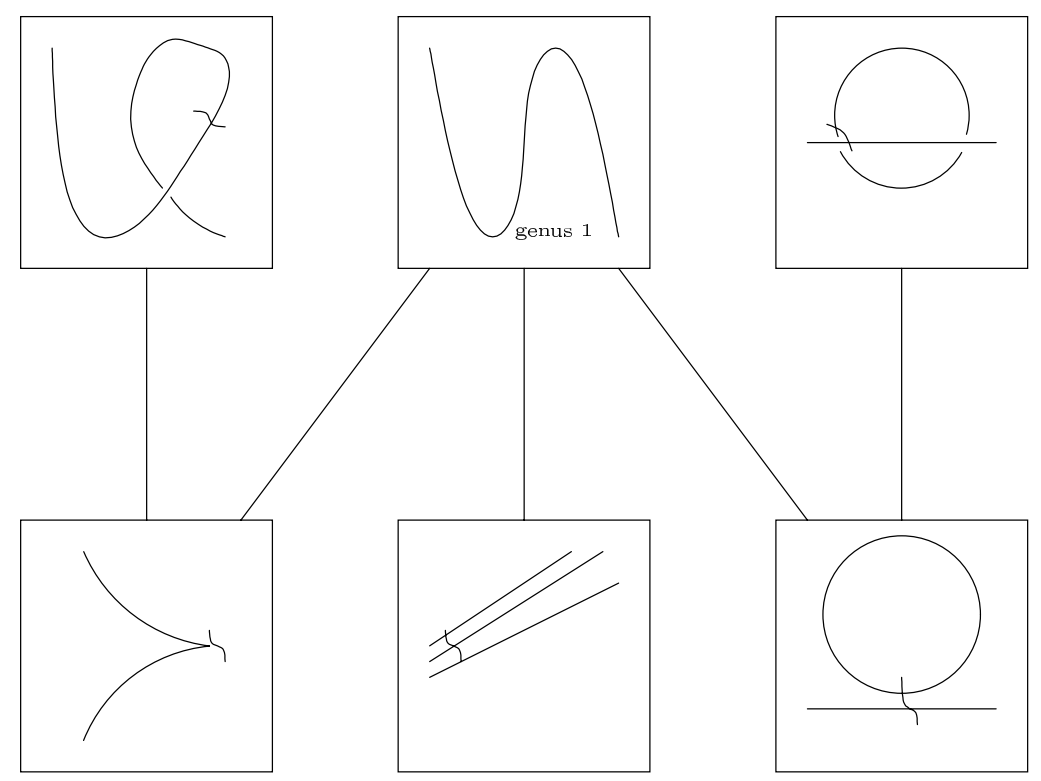

FiguRe 1. Irreducible components and other interesting loci in $\overline{\mathcal{M}}_{1}\left(\mathbb{P}^{2}, 3\right)$; the little squiggle in each panel represents a contracted genus-1 curve.

This construction also has a number of applications:

- Enumerative geometry of genus-1 curves via localization (extending results of [V1], for example adding tangencies).

- Gromov-Witten invariants in terms of enumerative invariants [Z1].

- The Lefschetz hyperplane property: effective computation of Gromov-Witten invariants of complete intersections [LZ2] (see also [LZ1] for the special case of the quintic threefold).

- An algebraic version of "reduced" Gromov-Witten invariants in symplectic geometry [Z1].

- Verification of the physicists' prediction BCOV] for genus-1 Gromov-Witten invariants of a quintic threefold in [Z3].

C. Fontanari $[\mathrm{F}]$ has recently shown that this desingularization has vanishing rational cohomology groups in odd degrees less than 10.

Before giving the construction, we motivate it by describing the geography of $\overline{\mathcal{M}}_{1, k}\left(\mathbb{P}^{n}, d\right)$. It is straightforward to show that $\overline{\mathcal{M}}_{1, k}\left(\mathbb{P}^{n}, d\right)$ is nonsingular on the locus where there is no contracted genus-1 (possibly nodal) curve (for example, the proof of [V1, Prop. 4.21] applies).

\section{Example: Plane cubics}

We first consider the case of $\overline{\mathcal{M}}_{1}\left(\mathbb{P}^{2}, 3\right)$; see Figure 1 . The main component generically corresponds to smooth plane cubics and has dimension 9. This is depicted in the upper-central panel of the figure. The remaining components must all contain a contracted genus- 1 curve, and we enumerate the possibilities. 
The contracted genus- 1 curve could meet one other curve, necessarily of genus 0 and mapping with degree 3 (see the upper-left panel of Figure11). The general such genus-0 map will have as its image a nodal cubic. This component of the moduli space has dimension 10: there is an 8-dimensional family of nodal cubics, plus a 1dimensional choice of where to "glue" the elliptic curve, plus a 1-dimensional choice of the $j$-invariant. Thus this locus cannot lie in the closure of the 9-dimensional main component.

Another possibility is that the contracted genus-1 curve could meet two other curves, one mapping with degree 2 and one mapping with degree 1 (see the upperright panel of Figure 11). This forms a 9-dimensional family: a 2-dimensional choice for the 2-pointed elliptic curve $\left(\operatorname{dim} \overline{\mathcal{M}}_{1,2}=2\right)$, plus a 2 -dimensional choice for the image of the contracted curve in the plane, plus a 4-dimensional choice of a conic through that point, plus a 1-dimensional choice of a line through that point. Again, for dimensional reasons, all such maps cannot lie in the 9-dimensional main component.

The final possibility involving a contracted elliptic component is if the contracted curve meets three other curves, each mapping with degree 1 (as lines). (See the lower-middle panel of Figure 1.) This family has dimension 8 (3 dimensions for the choice of a point in $\overline{\mathcal{M}}_{1,3}$, plus a 2 -dimensional choice of the image of this component in the plane, plus a 3-dimensional choice of the three lines through that point). Thus there is no dimensional obstruction for all such maps to lie in the (boundary of the) main component, and indeed they do.

One can extend this analysis to see where the components meet. The "one-tail component" meets the main component along the locus of maps where the genus0 degree 3 map has a cusp precisely where it meets the contracted elliptic curve (see the lower-left panel of Figure 1). The "two-tail component" meets the main component along the locus of maps where the conic and the line are tangent (see the lower-right panel of Figure 1). More generally, one can explicitly describe which genus-one stable maps are "smoothable" (i.e. lie in the main component):

Proposition. A genus-1 stable map $\pi: C \rightarrow \mathbb{P}^{n}$ is smoothable if and only if it is of one of the following two forms:

(i) $\pi$ contracts no genus-1 curve, or

(ii) if $E$ is the maximal connected genus-one curve contracted by $\pi$, and $E$ meets the rest of $C$ (i.e. $C^{\prime}=\overline{C-E}$ ) at the points $p_{1}, \ldots, p_{m}$, then $\pi\left(T_{C^{\prime}, p_{1}}\right), \ldots$, $\pi\left(T_{C^{\prime}, p_{m}}\right)$ must be a dependent set of vectors in $T_{\mathbb{P}^{n}, \pi(E)}$.

This readily follows from the same proof as in [V1, Lemma 5.9]. (More generally, one of the implications holds if $\mathbb{P}^{n}$ is replaced by a smooth target: if $C \rightarrow X$ is smoothable, then one of these two holds.)

Notice that this proposition "explains" the bottom row of Figure 1, if $E$ has "one tail" $(m=1)$, then $\pi\left(C^{\prime}\right)$ must have a cusp at that point for the map to be smoothable. If $E$ has "two tails" ( $m=2)$, then the two branches of $\pi\left(C^{\prime}\right)$ must be tangent at that point for the map to be smoothable. If $E$ has "three tails", then the three branches of $\pi\left(C^{\prime}\right)$ must be coplanar for the map to be smoothable - but this is automatic in $\mathbb{P}^{2}$. 


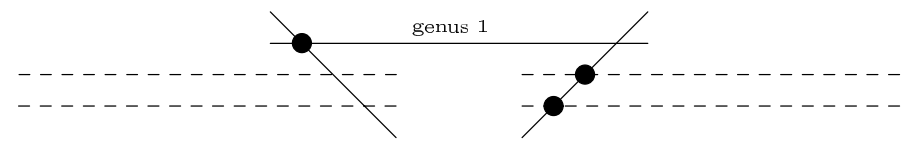

Figure 2. A map in two branches of the three-tail locus; the genus-one curve is indicated, the solid components are the contracted ones, and the large dots represent a choice of three nodes separating a genus-one contracted curve from the rest of the curve.

\section{The Desingularization PROCEDURE}

We finally describe the desingularization. We assume that $d>0$, since if $d=0$, then

$$
\overline{\mathcal{M}}_{1, k}\left(\mathbb{P}^{n}, d\right) \equiv \overline{\mathcal{M}}_{1, k} \times \mathbb{P}^{n},
$$

which is already smooth.

Define the $m$-tail locus of $\overline{\mathcal{M}}_{1, k}\left(\mathbb{P}^{n}, d\right)$ to be the closure of the subspace of $\overline{\mathcal{M}}_{1, k}\left(\mathbb{P}^{n}, d\right)$ consisting of the stable maps $\pi: C \rightarrow \mathbb{P}^{n}$ such that

(i) $C$ is a union of a smooth genus-one curve $E$ with a number of smooth genus-zero curves attached directly to $E$;

(ii) $E$ is contracted by $\pi$ and carries a total of $m$ marked and singular points;

(iii) none of the rational curves is contracted by $\pi$.

For example, in the 2-tail locus, the contracted elliptic curve might contain no marked points, and meet the rest of the curve in two points; or it might contain one marked point, and meet the rest of the curve in one point.

The $m$-tail locus is the union of a number of components, which we now describe. For each $m^{\prime} \in\{1, \ldots, d\}$, each partition $\mu$ of $d$ into $m^{\prime}$ parts, and each subset $S$ of $\{1, \ldots, k\}$ of size $m-m^{\prime}$, we have a subvariety (substack, really) corresponding to maps with a contracted elliptic curve $E$ containing the marked points $S$, and meeting $m^{\prime}$ genus-0 curves mapping with degrees corresponding to the partition $\mu$. These may or may not be components of $\overline{\mathcal{M}}_{1, k}\left(\mathbb{P}^{n}, d\right.$ ) (as we saw in the example of the cubics). Note that $E \subset C$ is not necessarily unique for a stable map $\pi: C \rightarrow \mathbb{P}^{n}$ in the $m$-tail locus; an example can be extracted from Figure 2.

Then the desingularization may be described as follows: blow up the one-tail locus, then the proper transform of the two-tail locus, etc. At each stage, we are blowing up along a smooth center.

We need to blow up in this particular order for the following reason. Figure 2 shows a map contained in the two-tail, three-tail, and four-tail locus. In fact, it is in "two branches" of the three-tail locus in the moduli space, corresponding to the two ways we can select three nodes separating a genus-one contracted curve from the rest of the curve. Thus the three-tail locus is not smooth at this point. Blowing up the two-tail locus will separate these two branches of the three-tail locus, and the proper transform of the three-tail locus is then smooth (at the points corresponding to this map).

We make a few observations.

First, this suggests that we should think of the one-tail locus as the "most singular locus", the two-tail locus as the "next most singular locus", and so on. This is perhaps opposite to the order one would expect. 
Second, note that blowing up a space $\left(\right.$ such as $\left.\overline{\mathcal{M}}_{1, k}\left(\mathbb{P}^{n}, d\right)\right)$ may be interpreted as removing the component ("blowing it out of existence"), and blowing up that component's scheme-theoretic intersection with the remainder of the space. More formally, if $X \cup Y$ is a scheme, with closed subschemes $X$ and $Y, \mathrm{Bl}_{X}(X \cup Y)$ is canonically isomorphic to $\mathrm{Bl}_{X \cap Y} Y$ by the universal property of blowing up. Hence we could equally well describe this construction as blowing up $\overline{\mathcal{M}}_{1, k}^{0}\left(\mathbb{P}^{n}, d\right)$ along the "one-tail locus" of this space, then the "two-tail locus", etc. (In this case, the first blowup, along the one-tail locus, does nothing, as this is already a Cartier divisor.) With this interpretation, at each stage we are still blowing up a space along a smooth center.

For example in the example of cubics, we remove the two non-main components (the upper-left and upper-right panels of Figure 1), blow up the locus corresponding to maps corresponding to the panel in the lower-right of Figure 1 (which is a Weil divisor, but not Cartier), and then blow up (the proper transform of) the locus corresponding to the panel in the lower-middle of Figure 1.

Third, this construction involves only the underlying curve and the information of which components are contracted. By making this precise, we are led to a candidate definition for more general target spaces. Let $\mathfrak{M}_{1, k}$ be the moduli space (Artin stack) of projective connected, nodal, genus-1, $k$-pointed nodal curves (over $\mathbb{C}$ ). Construct $\beta: \mathfrak{M}_{1, k}^{\prime} \rightarrow \mathfrak{M}_{1, k}$, where the points of $\mathfrak{M}_{1, k}^{\prime}$ are defined as projective, connected, nodal, genus-one curves with the additional information of a connected union of components of arithmetic genus-1 (possibly empty) that is declared to be contracted. Then $\beta$ is locally (on the source) an isomorphism, but is not separated. The forgetful morphism $\overline{\mathcal{M}}_{1, k}\left(\mathbb{P}^{n}, d\right) \rightarrow \mathfrak{M}_{1, k}$ naturally factors through $\mathfrak{M}_{1, k}^{\prime}$. If

$\widetilde{\mathfrak{M}^{\prime}}{ }_{1, k}$ is the blowup of $\mathfrak{M}_{1, k}^{\prime}$ along the one-tail locus, the proper transform of the two-tail locus, etc., then

$$
\overline{\mathcal{M}}_{1, k}\left(\mathbb{P}^{n}, d\right) \times_{\mathfrak{M}_{1, k}^{\prime}} \widetilde{\mathfrak{M}^{\prime}}{ }_{1, k}
$$

contains $\widetilde{\mathcal{M}}_{1, k}\left(\mathbb{P}^{n}, d\right)$ as an irreducible component. If $X$ is a complex projective manifold, one can similarly define $\widetilde{\mathcal{M}}_{1, k}(X, \beta)$ as the union of components of

$$
\overline{\mathcal{M}}_{1, k}(X, \beta) \times_{\mathfrak{M}_{1, k}^{\prime}} \widetilde{\mathfrak{M}_{1, k}^{\prime}}
$$

generically mapping to $\mathfrak{M}_{1, k}$ (i.e. corresponding to maps with smooth source). (We have no reasonable modular interpretation of $\widetilde{\mathcal{M}}_{1, k}(X, \beta)$ in general; taking the closure is an awkward construction moduli-theoretically.) Via the exact sequence for the tangent-obstruction theory of $\overline{\mathcal{M}}_{1, k}(X, \beta)$ in terms of that of $\mathfrak{M}_{1, k}$ and $H^{i}\left(C, \pi^{*} T_{X}\right)$, one can endow (10) with a natural virtual fundamental class. We expect this to lead to an algebraic theory of "reduced genus-1 Gromov-Witten invariants"; cf. [Z1].

\section{REFERENCES}

[BCOV] M. Bershadsky, S. Cecotti, H. Ooguri, and C. Vafa, Holomorphic anomalies in topological field theories, Nucl. Phys. B 405 (1993), 279-304. MR1240687 (94j:81254)

[F] C. Fontanari, Towards the cohomology of moduli spaces of higher genus stable maps, preprint 2006, math.AG/0611754.

[LZ1] J. Li and A. Zinger, On the genus-one Gromov-Witten invariants of a quintic threefold, preprint 2004, math.AG/0406105.

[LZ2] J. Li and A. Zinger, On the genus-one Gromov-Witten invariants of complete intersections, preprint 2005, math.AG/0507104. 
[P] R. Pandharipande, Intersections of $\overline{\mathbb{Q}}$-divisors on Kontsevich's moduli space $\bar{M}_{0, n}\left(\mathbb{P}^{r}, d\right)$ and enumerative geometry, Trans. Amer. Math. Soc. 351 (1999), no. 4, 1481-1505. MR:1407707 (99f:14068)

[V1] R. Vakil, The enumerative geometry of rational and elliptic curves in projective space, J. Reine Angew. Math. (Crelle's Journal) 529 (2000), 101-153. MR.1799935 (2001j:14072)

[V2] R. Vakil, Murphy's Law in algebraic geometry: Badly-behaved moduli deformation spaces, Invent. Math. 164 (2006), 569-590. MR2227692 (2007a:14008)

[VZ] R. Vakil and A. Zinger, A desingularization of the main component of the moduli space of genus-one stable maps to projective space, preprint 2006, math.AG/0603353v1.

[Z1] A. Zinger, Reduced genus-one Gromov-Witten invariants, preprint 2005, math.SG/0507103.

[Z2] A. Zinger, Intersections of tautological classes on blowups of moduli spaces of genus-one curves, preprint 2006, math.AG/0603357.

[Z3] A. Zinger, The reduced genus-one Gromov-Witten invariants of Calabi-Yau hypersurfaces, preprint 2007, math/0705.2397.

Department of Mathematics, Stanford University, Stanford, California 94305-2125

E-mail address: vakil@math.stanford.edu

Department of Mathematics, SUny Stony Brook, Stony Brook, New York 11794-3651

E-mail address: azinger@math.sunysb.edu 